\title{
An evaluation of serum homocysteine level as a biological marker in Oral sub mucous fibrosis patients in a western setting of Uttar Pradesh, India
}

\author{
Dr. Prabhakar Singh Bais, Mrs. Priyanka chauhan, Dr. Shishir Mohan \\ Prof. \& Head Dept. of Biochemistry K.D.Dental college \& Hospital-Mathura( U.P.) \\ Sr. Lecturer Dept. of Pathology \& Microbiology K.D.Dental college \& Hospital-Mathura( U.P.) \\ Prof. \& HeadDept. of oral \& Maxillofacial surgeoryK.D.Dental college \& Hospital-Mathura (U.P.)
}

\begin{abstract}
:
Objective: The aim of the present study was to evaluate the serum homocysteine level as a biological marker in Oral sub mucous fibrosis patients.

Methods: This was a cross-sectional study conducted in a tertiary care hospital. A total of 50 patients diagnosed with oral sub mucous fibrosis not taking treatment clinically and histologically diagnosed were included in the study. Patients suffering from cardiovascular diseases, osteoporosis, dementia, alzheimer's disease, diabetes, Oral cancer, blood cancer, lung cancer, breast cancer and other malignancies were excluded from the study.
\end{abstract}

Results: The average age of the patients was 34.9 ( \pm 9.00$)$ years. Majority were males (94\%). More than half (54\%) of the patients were in stage II followed by III (36\%) and IV (10\%). The overall homocysteine level was 24.1 ( \pm 3.3$) \mu \mathrm{mol} / \mathrm{lit}$ (Table not shown). The level of homocysteine was similar male and female patients. No females were in stage III. The homocysteine level was higher among the patients of stage IV (31.02 \pm 6.33$)$ than

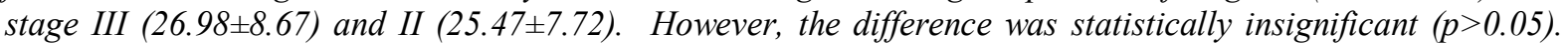
There was no significant difference in the level of homocysteine by gender and clinical staging.

Conclusion: A longitudinal study of larger groups of patients with OSMF is necessary, as are lengthier followups, to define the real role of serum homocysteine.

\section{Introduction}

Oral submucous fibrosis (OSMF) is a chronic disease of insidious onset sometimes preceded by vesicle formation or stomatitis and always associated with juxtaepithelial inflammatory reaction and fibroelastic changes of lamina propria with epithelial atrophy. A condition resembling OSMF was described as early as "600" BC by Sushruta and it was named as "VIDARI" having features of progressive narrowing of mouth, depigmentation of oral mucosa and pain on taking food. The present nomenclature was coined by Joshi from Bombay ${ }^{1}$ and was designated as "submucous fibrosis of palate and pillars". Overall prevalence of oral submucous fibrosis in India is about $0.5 \%$ with a range of $0.2-1.2 \%$ in different regions of country. The exact etiology of OSMF is not well understood. Various factors are being studied, such as genetic, autoimmune, nutritional and environmental agents. Among the environmental agents different oral habits are observed like intake of spicy food, chewing of betal nut, betal quid and allied preparations (pan masala, gutka, kharra, etc.). As it is a disease of unknown etiology, till date no satisfactory treatment has been described in literature for OSMF.

Homocysteine is an intermediary aminoacid formed during the conversion of methionine to cysteine. Elevated total plasma level of aminoacid homocysteine has been identified as an independent risk factor of arteriosclerosis involving coronary, cerebral , and peripheral arteries. Hyperhomocysteinemia is suggested to be an independent risk factor for premature vascular disease ${ }^{2}$, myocardial infarction ${ }^{3}$, and stroke ${ }^{4}$. Furthermore, raised homocysteine levels have been suggested as a risk factor for non-arteritic anterior ischemic optic neuropathy ${ }^{5}$. In our best knowledge, there is no study in which the level of homocysteine have been assessed among OSMF patients.

The aim of the present study was to evaluate the serum homocysteine level as a biological marker in Oral sub mucous fibrosis patients.

\section{Material And Methods}

This was a cross-sectional study conducted in a tertiary care hospital after ethical approval from the ethical committee of the hospital. A written informed consent was taken from each of the patient before enrolling in the study. A total of 50 patients diagnosed with oral sub mucous fibrosis not taking treatment clinically and histologically diagnosed were included in the study. Patients suffering from cardiovascular 
diseases, osteoporosis, dementia, alzheimer's disease, diabetes, Oral cancer, blood cancer, lung cancer, breast cancer and other malignancies were excluded from the study.

\section{Clinical Examination}

The subjects were made to sit comfortably on dental chair with artificial illumination. Wearing sterile hand gloves and mouth mask, the subjects were examined under artificial illumination. The clinical examination was carried out by adopting methods of Khanna and Andrade ${ }^{6}$ and relevant data were recorded in the Performa. A clinical diagnosis of oral sub mucous fibrosis was made on relevant history and thorough clinical examination of oral cavity. The history obtained from patient included the chief complaint, history of present illness, habits like gutka chewing; betel nut chewing and quid placement was recorded. The clinical examination included a detailed clinical inspection of oral cavity to note the presence of oral sub mucous fibrosis based on clinical stages proposed by Khanna and Andrade ${ }^{6}$ for clinical course of the oral sub mucous fibrosis.

\section{Biopsy}

Incisional and punch biopsy is a process of surgical removal of tissue from a patient for pathological examination. In our study we performed this procedure for all 50 patients diagnosed clinically with OSMF in order to confirm the clinical diagnosis. Guidelines given by Oliver et al ${ }^{7}$ were followed for biopsy procedure.

All study group patients under went biopsy procedure on same day. Basic blood investigations like hemoglobin $\%$, total leukocyte count, total RBC count, bleeding time, clotting time were carried out for all patients before biopsy procedure. The patients were seated comfortably in dental chair and draped. Local anesthesia was obtained by giving a regional block with $2 \%$ lignocaine with 1:80,000adrenaline.

Once anesthesia was obtained a wedge of tissue involving the lesion and normal tissue was incised using $5 \mathrm{~mm}$ punch kit and preserved in $10 \%$ formalin and sent the tissue specimen for Histo-pathological examination. Homeostasis was obtained by guaze pack and sutures were placed wherever necessary with 3-0 interrupted silk suture. Post-operatively broad spectrum antibiotics Amoxicillin 500mg thrice a day for five days and an Ibubrufen 400mg BD for 5 days was prescribed.

Patients were advised to avoid hot spicy food for a day and to take lot of liquids, soft diet, warm saline rinses from the next subsequent day and do not touch the excised area. They were advised to quit all the deleterious habits. In cases where sutures were placed, theywere recalled after a week for suture removal.

\section{Measurement of Homocysteine}

Blood samples were collected by vein puncture in vacuette. The patient's forearm was rested on laboratory table comfortably. The ante-cubital fossa was exposed and Velkret tourniquet was applied about half an inch to two inches above ante-cubital fossa. The area was rendered aseptic with $70 \%$ ethyl alcohol and using $5 \mathrm{ml}$ sterile disposable syringe and 23 guaze needles a needle puncture was made and maneuvered to enter the anti-cubital vein and $5 \mathrm{ml}$ of blood was drawn. Thenthe tourniquet was relieved and needle was removed simultaneously. Spirit soaked cotton was placed on site of needle puncture on forearm and instructions were given to apply finger pressure for about five minutes and dispose the cotton.

\section{Serum separation and storage}

Blood from the syringe was transferred to vacoutainer and was allowed to clot for 10-15 minutes. Vacoutainer were centrifuged at 4000rpm for 15 minutes and serum was separated from the clotted blood. Separated serum was transferred to fresh vacoutainer and sent for estimation of Homocysteine to thelaboratory (Immuno-Diagnostics Pvt Ltd an ISO: 9001-2008 certified Lab. Each sample was analyzed by Immunoassay technique to find the Homocysteine level in the serum.

\section{Statistical analysis}

The results are presented in mean $\pm \mathrm{SD}$ and percentage. The One Way analysis of Variance was used to compare the homocysteine level among clinical staging. The $\mathrm{p}$-value $<0.05$ is being considered as significant. All the analysis was carried out by using SPSS 16.0 version.

\section{Results}

The average age of the patients was $34.9( \pm 9.00)$ years. Majority were males $(94 \%)$. More than half $(54 \%)$ of the patients were in stage II followed by III (36\%) and IV (10\%) (Table-1).

The overall homocysteine level was 24.1 ( \pm 3.3$) \mu \mathrm{mol} / \mathrm{lit}$ (Table not shown). The level of homocysteine was similar male and female patients (Fig.1).

Table-2 describes the comparison of homocysteine ( $\mu \mathrm{mol} / \mathrm{lts})$ with clinical staging of OSMF. No females were in stage III. The homocysteine level was higher among the patients of stage IV (31.02 \pm 6.33$)$ than 
stage III (26.98 \pm 8.67$)$ and II $(25.47 \pm 7.72)$. However, the difference was statistically insignificant $(\mathrm{p}>0.05)$. There was no significant difference in the level of homocysteine by gender and clinical staging.

\section{Discussion}

Oral submucous fibrosis (OSF) is an insidious chronic condition that can affect any part of the oral cavity and sometimes even the pharynx. Although occasionally preceded by and /or associated with vesicle formation, OSF is always associated with epithelial inflammatory reaction followed by fibroelastic changes to the lamina propria with and causing trismus and inability to eat ${ }^{8}$.

epithelial atrophy leading to stiffness of the mucosa

Serum homocysteine level is a sensitive indicator of folate status. In fact, folate deficiency is often associated with hyperhomocysteinemia, and folate intake can reduce the homocysteine level ${ }^{9}$. Folate deficiency aids the incorporation of uracil into the DNA, which can lead to DNA instability. High levels of homocysteine are associated with colorectal cancer ${ }^{10}$, as well as uterine cervical cancer ${ }^{11}$. Alterations in the methionine cycle are also thought to be involved in breast ${ }^{12}$ and pancreatic cancer ${ }^{13}$. Although moderate folate deficiency alone might not be mutagenic in vivo, it seems to interact with other risk factors, either environmental or genetic, in promoting tumor progression ${ }^{14}$.

The present study was aimed to evaluate the serum homocysteine level as a biological marker in Oral sub mucous fibrosis patients. In our best knowledge, this is the first study evaluating the level of serum homocysteine among the patients of oral sub mucous fibrosis. In the present study, the overall homocysteine level was $24.1( \pm 3.3) \mu \mathrm{mol} / \mathrm{lit}$. The level of homocysteine was similar among male and female patients. No females were in stage III. The homocysteine level was higher among the patients of stage IV than stage III and II. However, the difference was statistically insignificant $(\mathrm{p}>0.05)$. There was no significant difference in the level of homocysteine by gender and clinical staging.

\section{Conclusion}

In conclusion, we found an interesting association between serum homocysteine and clinical staging of OSMF, however, insignificant. A longitudinal study of larger groups of patients with OSMF is necessary, as are lengthier follow-ups, to define the real role of serum homocysteine.

Table-1: General characteristics of the patients

\begin{tabular}{|l|c|}
\hline & $\mathbf{n}=\mathbf{5 0}$ \\
\hline Age in years, mean \pm SD & $34.9 \pm 9.00$ \\
\hline Gender, no. (\%) & $47(94.0)$ \\
\hline Male & $3(6.0)$ \\
\hline Female & $0(0.0)$ \\
\hline Clinical staging of OSMF & $27(54.0)$ \\
\hline I & $18(36.0)$ \\
\hline II & $5(10.0)$ \\
\hline III & \\
\hline IV & \\
\hline
\end{tabular}

Table-2: Comparison of homocysteine ( $\mu \mathrm{mol} / \mathrm{lts})$ with clinical staging of OSMF

\begin{tabular}{|c|c|c|c|c|c|}
\hline \multirow{2}{*}{ Gender } & \multicolumn{5}{|c|}{ Clinical staging } \\
\hline & I & II & III & IV & p-value \\
\hline Males & - & $25.88 \pm 7.84$ & $26.98 \pm 8.67$ & $32.93 \pm 5.41$ & 0.276 \\
\hline Females & - & $20.35 \pm 4.17$ & - & $23.40 \pm 0.00$ & 0.657 \\
\hline Total & & $25.47 \pm 7.72$ & $26.98 \pm 8.67$ & $31.02 \pm 6.33$ & 0.355 \\
\hline
\end{tabular}




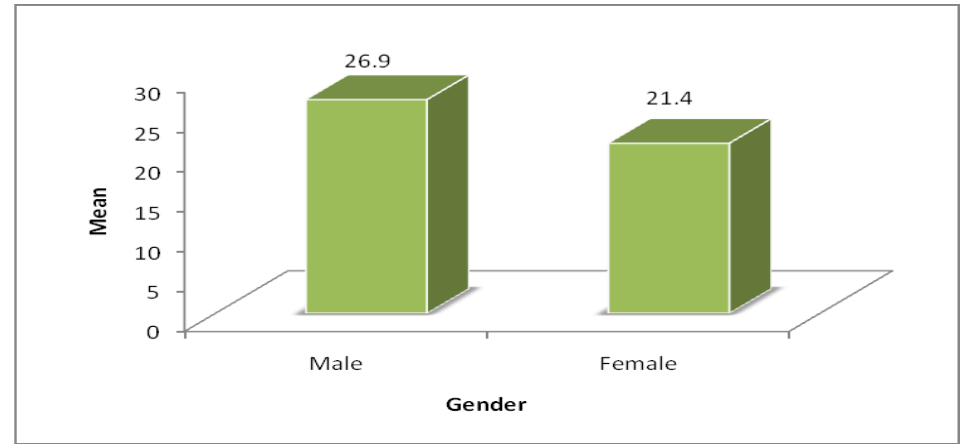

Fig.1: Comparison of homocysteine ( $\mu \mathrm{mol} / \mathrm{lts})$ with gender

\section{References}

[1]. Akbar M. Oral submucous fibrosis - A clinical study. J Indian Dent Assoc 1976;48:363-75.

[2]. Clarke R,Daly L, Robinson K, Naughten E et al.Hyperhomocysteinemia: an independent risk factor for vascular disease.N Engl J Med 1991;324:1149-1155.

[3]. Stampfer MJ, Malinow MR, Willett WC,Newcomer LM et al. A prospective study of plasma homocysteine and risk of myocardial infarction in US physicians.JAMA 1992;268:877-881.

[4]. Perry IJ, Refsum H, Morris RW et al. Prospective study of serum total homocysteine concentration and risk of stroke in middle -aged British men. Lancet 1995;346:1395-1398.

[5]. Kawasaki A,Purvin VA,Burgett RA. Hyperhomocysteinemia in young patients with non-arteritic anterior ischemic optic neuropathy. Br J Ophthalmol 1999; 83:1287-90.

[6]. Khanna JN, Andrade NN et al. Oral Submucous fibrosis: A new concept in surgical management - Report of 100 cases. International Journal of Oral and Maxillofacial surgery 1995;24:433-439.

[7]. Oliver RJ, Sloan P et al. Oral Biopsies: methods and application. Br Dent J 2004; 196(6):329-33.

[8]. Pindborg JJ, Sirsat SM :Oral submucous fibrosis. Oral Surgery Oral Medicine \& Oral Pathology, 22: 764-779,1966

[9]. Selhub J, Jacques PF, Wilson PW, et al. Vitamin status and intake as primary determinants of homocysteinemia in an elderly population. JAMA. 1993;270 (22):2693-2698.

[10]. Kato I, Dnistrian AM, Schwartz M, et al. Serum folate, homocysteine and colorectal cancer risk in women: a nested case-control study. Br J Cancer. 1999; 79(11-12):1917-1921.

[11]. Alberg AJ, Selhub J, Shah KV, et al. The risk of cervical cancer in relation to serum concentration of folate, vitamin B12 and homocysteine. Cancer Epidemiol Biomarkers Prev. 2000;9(7):761-764.

[12]. Wu K, Helzlsouer KJ, Comstock GW, et al. A prospective study on folate, B12, and pyridoxal 5_-phosphate (B6) and breast cancer. Cancer Epidemiol Biomarkers Prev. 1999;8(3):209-217.

[13]. Stolzenberg-Solomon RZ, Albanes D, Nieto FJ, et al. Pancreatic cancer risk and nutrition-related methyl-group availability indicators in male smokers. J Natl Cancer Inst. 1999;91(6):535-541.

[14]. Almadori G, Bussu F, Galli J, et al. Serum levels of folate, homocysteine, and vitamin B12 in head and neck squamous cell carcinoma and in laryngeal leukoplakia. Cancer. 2005;103(2):284-292. 